CONCISE REPORT

\title{
Radiographic progression in children with polyarticular juvenile rheumatoid arthritis: a pilot study
}

\author{
T Mason, A M Reed, A M Nelson, K B Thomas
}

Ann Rheum Dis 2005;64:491-493. doi: 10.1136/ard.2003.017053

\begin{abstract}
Objective: To assess disease progression on hand/wrist $x$ rays from children with polyarticular juvenile rheumatoid arthritis.

Methods: Initial and subsequent films of 13 white children (10 girls) were read blind by a paediatric radiologist for the presence of joint space narrowing (JSN), erosions, and relative carpal length ( $R C L)$.

Results: One child had subcutaneous nodules; one (of 11) was rheumatoid factor positive; six were ANA positive. Median age at diagnosis was 10.7 years (2.5 to 15.9). Median number of involved joints (swelling, pain, or decreased range of motion) at diagnosis was 16 (6 to 33). Four initial $x$ rays had either erosions or JSN. Subsequent $x$ rays were done at (median) 13.3 (8.3 to 24.9) months after initial $x$ rays. One of 10 subsequent $x$ rays had shortened $\mathrm{RCL}$, and six of 13 were worse than the initial ones. Four of these developed new erosions, one had increased number of erosions, and one developed new JSN.

Conclusions: About half the children with polyarticular juvenile rheumatoid arthritis will have evidence of radiographic progression within two years after diagnosis. Thus newly diagnosed children are at high risk of substantial joint destruction and potential disability, emphasising the need for prompt treatment.
\end{abstract}

\footnotetext{
J
} uvenile rheumatoid arthritis is a common chronic illness of childhood with an incidence of approximately 1 per 10000 children per year and a prevalence of about 1 per 1000 children. ${ }^{1}$ There are three distinct subtypes, based on the clinical presentation of the disease. ${ }^{2}$ Polyarticular juvenile rheumatoid arthritis is defined by the presence of arthritis in five or more joints with the absence of systemic features within the first six months of the illness.

Previous studies have shown that the results of $x$ rays of the hands and wrists have correlated well with other clinical outcome measures in adults with rheumatoid arthritis. ${ }^{34}$ Methods of assessing changes in radiographs in adults with this disease include the Larsen method ${ }^{5}$ and the Sharp score. ${ }^{6}$ General indices for assessing radiographic damage of patients with rheumatoid arthritis include osteopenia, joint space narrowing, and cortical erosions. ${ }^{7}$ Erosive disease in patients with rheumatoid arthritis suggests a high likelihood of joint damage and a poor clinical outcome. ${ }^{8}$ Polyarticular juvenile rheumatoid arthritis shares many clinical features with adult rheumatoid arthritis, including frequent involvement of the hands and wrists and a progressive and potentially destructive natural history.

Poznanski et al described a method for assessing $x$ ray damage in hands and wrists of children by assessing the relative length of the carpus (RCL). ${ }^{9}$ This method was recently used in another study of clinical outcomes in young children with juvenile idiopathic arthritis. ${ }^{10}$ In our pilot study, we evaluated the hand/wrist $x$ rays in a small cohort of children with newly diagnosed polyarticular juvenile rheumatoid arthritis who had a subsequent hand/wrist $x$ rays taken at least eight months after the initial films. We used both RCL assessment and the indices used for assessing damage in rheumatoid arthritis.

\section{METHODS}

We studied 13 cases of newly diagnosed polyarticular juvenile rheumatoid arthritis from January 1986 to December 2000. All had had $x$ rays of the hands and wrists at their initial visit, and had a second set at least eight months later. The study radiologist was given the initial and subsequent $x$ rays and was asked to interpret them. She recorded her findings on a preprepared data collection form, commenting on the presence of soft tissue swelling, periarticular osteopenia, joint space narrowing, and pericortical erosions. She was blinded to any clinical information about the subjects and she was not able to distinguish between the initial and subsequent $x$ rays. She also undertook measurements of the radiocarpal length (RM) and length of the second metacarpal (M2) on each $x$ ray. If the carpal physes (growth plates) were closed on either the initial or the subsequent $x$ rays, then the RCL was not calculated. These measurements were plotted on the curve derived by Poznanski et al ${ }^{9}$ to assess RCL for age.

Radiographic worsening ("progression") was assessed by the question: "Is one of the $x$ rays worse than the other?" If the radiologist felt the initial hand/wrist $x$ ray was worse than the subsequent one, the subject was not assessed as having radiographic progression (this did not occur in this study). If a subject had radiographic progression, the radiologist also indicated whether joint space narrowing had worsened, the number of erosions had increased, or whether both had occurred. "Shortening" of RCL was defined as any decrease in RCL on the subsequent film.

\section{RESULTS}

All 13 children were white, and 10 were female. Median age at the time of diagnosis was 10.7 years (range 2.5 to 15.9), and the median duration of hand/wrist symptoms at the time of diagnosis was 6.0 months (2 to 11 ). All had polyarticular onset and none had extended oligoarticular disease. The median number of involved joints (decreased range of movement (ROM), painful movement, or swelling) at the time of diagnosis was 16 (6 to 33). At the time of diagnosis, one had subcutaneous nodules. One of 11 had raised serum rheumatoid factor (RF), and six of 13 had serum antinuclear antibodies (ANA) in a titre of more than $1: 40$ or its equivalent. All the initial hand/wrist $x$ rays were done within six weeks of the date of diagnosis. The clinical data are summarised in table 1 .

Abbreviations: DMARD, disease modifying antirheumatic drug; JSN, joint space narrowing; $\mathrm{RCL}$, relative carpal length 
Table 1 Clinical features of children with polyarticular juvenile rheumatoid arthritis and radiographic progression

\begin{tabular}{|c|c|c|c|c|c|c|}
\hline & Sex & $\begin{array}{l}\text { Age at diagnosis } \\
\text { (y) }\end{array}$ & $\begin{array}{l}\text { Joint count at } \\
\text { diagnosis }\end{array}$ & RF+ & ANA + & Nodules \\
\hline \multicolumn{7}{|c|}{ Subjects with progression } \\
\hline 1 & $\mathrm{~F}$ & 14.7 & 19 & 0 & + & 0 \\
\hline 2 & $M$ & 13.5 & 12 & 0 & + & 0 \\
\hline 3 & $\mathrm{~F}$ & 11.6 & 12 & $\mathrm{~N} / \mathrm{A}$ & 0 & 0 \\
\hline 4 & $\mathrm{~F}$ & 10.6 & 6 & + & 0 & + \\
\hline 5 & $\mathrm{~F}$ & 8.3 & 24 & 0 & 0 & 0 \\
\hline \multirow[t]{2}{*}{6} & $\mathrm{~F}$ & 15.9 & 33 & 0 & 0 & 0 \\
\hline & & Median $=12.6$ & Median $=15.5$ & & & \\
\hline \multicolumn{7}{|c|}{ Subjects with no progression } \\
\hline 7 & $\mathrm{~F}$ & 3.3 & 26 & 0 & 0 & 0 \\
\hline 8 & $\mathrm{~F}$ & 10.7 & 10 & 0 & + & 0 \\
\hline 9 & $M$ & 13.7 & 24 & 0 & 0 & 0 \\
\hline 10 & $\mathrm{~F}$ & 2.5 & 20 & $\mathrm{~N} / \mathrm{A}$ & + & 0 \\
\hline 11 & $\mathrm{~F}$ & 5.4 & 12 & 0 & + & 0 \\
\hline 12 & $\mathrm{~F}$ & 15.9 & 16 & 0 & 0 & 0 \\
\hline \multirow[t]{2}{*}{13} & $M$ & 9.2 & 14 & 0 & + & 0 \\
\hline & & Median $=9.2$ & Median $=16$ & & & \\
\hline
\end{tabular}

Progression $=$ increase in JSN or number of erosions on subsequent hand/wrist $x$ ray.

No progression $=$ no increase in JSN or number of erosions on subsequent hand/wrist $x$ ray.

Joint count at diagnosis = number of joints involved (joint swelling, pain on movement, tender joint, limited ROM) at time of diagnosis.

Nodules $=$ presence of subcutaneous nodules at time of diagnosis.

$\mathrm{ANA}+$, raised titre of antinuclear antibodies at time of diagnosis; JSN, joint space narrowing; $\mathrm{RF}+$, raised titre of serum rheumatoid factor at time of diagnosis; ROM, range of movement; $y$, years.

Thirteen sets of initial hand/wrist $x$ rays were studied. Four of these had erosions or joint space narrowing. The median time between initial and subsequent $x$ rays was 13.3 months (range 8.3 to 24.9 ). In one subject, erosions did not progress from the initial hand/wrist $x$ ray.

Six subjects $(46 \%)$ had evidence of radiographic progression. Of the six that progressed, five had erosive disease on the subsequent $x$ rays (all of these had joint space narrowing). Four of the five with erosions on subsequent $x$ rays had developed new erosions, and one had an increased number of erosions. One of the six that progressed developed joint space narrowing without erosions. All the erosions were either in the carpal or metacarpophalangeal joints. Those that progressed had a longer delay in initiation of disease modifying antirheumatic drug (DMARD) treatment.

Two of the children had closed carpal physes at diagnosis and one had closed physes on the subsequent hand/wrist $x$ rays, so an accurate assessment of RCL was not possible in these three subjects. Of the 10 remaining subjects, one had a shortened RCL on subsequent $x$ ray. Four of the five who progressed and had assessment of RCL did not have shortening. These radiographic data are summarised in table 2 .

\section{DISCUSSION}

This was a pilot study to evaluate the rate of radiographic progression of hand/wrist $x$ rays in children with newly

Table 2 Radiographic progression and disease modifying antirheumatic drug use

\begin{tabular}{|c|c|c|c|c|c|}
\hline & Erosion or JSN & Shortened RCL & Erosion location & $\begin{array}{l}\text { Time between } \\
\boldsymbol{x} \text { rays }(\mathbf{m})\end{array}$ & DMARD delay (m) \\
\hline \multicolumn{6}{|c|}{ Subjects with progression } \\
\hline 1 & Y & N/A & $C, M$ & 14.6 & 0.0 \\
\hline 2 & $Y$ & Y & $C, M$ & 11.0 & 5.4 \\
\hline 3 & Y & $\mathrm{N}$ & 0 & 12.1 & 14.4 \\
\hline 4 & $Y$ & $\mathrm{~N}$ & $M$ & 20.1 & 14.0 \\
\hline 5 & $Y$ & $\mathrm{~N}$ & $C, M$ & 22.6 & 9.6 \\
\hline \multirow[t]{2}{*}{6} & Y & N/A & C & 24.9 & 0.0 \\
\hline & $100 \%$ & $25 \%$ & & Median $=17.4$ & Median $=7.5$ \\
\hline \multicolumn{6}{|c|}{ Subjects with no progression } \\
\hline 7 & $\mathrm{~N}$ & $\mathrm{~N}$ & 0 & 13.3 & 0.0 \\
\hline 8 & $\mathrm{~N}$ & $\mathrm{~N}$ & 0 & 8.3 & 8.3 \\
\hline 9 & $\mathrm{~N}$ & $\mathrm{~N}$ & 0 & 11.6 & 5.5 \\
\hline 10 & $\mathrm{~N}$ & $\mathrm{~N}$ & 0 & 18.2 & 1.6 \\
\hline 11 & $\mathrm{~N}$ & $\mathrm{~N}$ & 0 & 11.2 & 1.7 \\
\hline 12 & $Y$ & N/A & C & 19.6 & 0.0 \\
\hline \multirow[t]{2}{*}{13} & $N$ & $N$ & 0 & 12.0 & 0.0 \\
\hline & $16 \%$ & $0 \%$ & & Median $=12$ & Median $=1.6$ \\
\hline
\end{tabular}

Progression $=$ increase in JSN or number of erosions on subsequent hand/wrist $x$ ray. Erosion or JSN = erosion or joint space narrowing on subsequent hand/wrist $x$ ray. Shortened $R C L=R C L$ on subsequent $x$ ray less than on initial $x$ ray.

Erosion location $=$ location of erosions on subsequent $x$ ray $(0$, no erosions).

DMARD delay = time between date of diagnosis of polyJRA and first dose of disease modifying medication (including hydroxychloroquine, sulfasalazine, oral or parenteral gold, methotrexate).

$\mathrm{C}$, carpal; DMARD, disease modifying antirheumatic drug; I, proximal or distal interphalangeal, JSN, joint space narrowing; $m$, months; $M$, metacarpophalangeal; $N$, no; N/A, not assessed; $R C L$, relative carpal length; $Y$, yes. 
diagnosed polyarticular juvenile rheumatoid arthritis. About $50 \%$ of adult patients with rheumatoid arthritis may have erosions within three years of their diagnosis. ${ }^{11}$ The data from our study are consistent with this observation, as nearly half our cohort of children had erosive disease within two years of diagnosis.

We have previously reported our experience in assessing the relative frequency of radiographic abnormalities-such as periarticular osteopenia, joint space narrowing, and erosions-in a cohort of children with newly diagnosed polyarticular juvenile rheumatoid arthritis. ${ }^{12}$ In that cohort of nearly 20 children, over a quarter had either erosions or joint space narrowing at the time of diagnosis.

Only one of our subjects had "shortening" of RCL. This child also had radiographic progression. As most of the subjects with radiographic progression did not have shortening of the RCL, perhaps assessment of this variable is a less sensitive indicator of radiographic change than assessment of joint space narrowing and erosions in an older cohort of children with polyarticular disease.

We attempted to identify indices that would distinguish those who progressed from those who did not, but the small number of subjects in the study limited the generalisability of the findings. The responses to the various therapeutic interventions would clearly affect the likelihood of progression. Also, differences in the time between the initial and subsequent $x$ rays may also account for some of the variation between the groups with respect to progression and shortening. We could not rule out a selection bias favouring cases with more clinically severe disease, who might have been more likely to have had hand/wrist $x$ rays. However, these pilot data suggest that the rate of radiographic progression in polyarticular juvenile rheumatoid arthritis is significant, and further study is indicated.

A paper by Oen et al showed that joint space narrowing but not erosions correlated with clinical outcome measures in children with juvenile rheumatoid arthritis after several years of disease. ${ }^{13}$ Our study differs substantially from that cross sectional study, as ours looked at radiographic progression in newly diagnosed polyarticular disease.

A recent study by van Rossum et al described a method for evaluating joint radiographs in children with juvenile idiopathic arthritis. ${ }^{14}$ In that study of children with all subtypes of the disease the correlation of radiographic findings with clinical findings was also good.

Another recent paper, by Magni-Manzoni et al, showed a strong relation between the baseline Poznanski score of the wrist and the radiographic and clinical outcomes in a cohort of nearly 100 children with juvenile idiopathic arthritis. ${ }^{10}$ This group was quite young, with a median age of 4.6 years, and included systemic onset, extended oligoarticular, and polyarticular disease. This very important study showed that serial radiographic assessment correlates with important clinical outcome (as assessed, for example, by the child health assessment questionnaire (CHAQ)). Their study differed from ours in several ways. Their cohort was quite young and included systemic onset and extended oligoarticular disease, which were excluded from our cohort. Also, our subjects had $x$ rays of the hands and wrists, not just the wrists. Finally, the median duration of disease at baseline was 1.1 years, while our cohort had baseline studies at diagnosis. It may be that multiple methods of assessing these $x$ rays will be necessary, to account for the limitations of the Poznanski score and for maturing carpal bones.

Our data suggest that children with newly diagnosed polyarticular juvenile rheumatoid arthritis are at significant risk of radiographic progression within the first two years of the disease, as in adults with rheumatoid arthritis. The data also support the need for timely diagnosis and initiation of treatment in this disease. Prospective studies using these techniques of radiographic evaluation could assess the degree to which serial hand/wrist $x$ rays correlate with the clinical outcome.

\section{Authors' affiliations}

T Mason, A M Reed, Division of Rheumatology, Mayo Clinic College of Medicine, Rochester, Minnesota, USA

A M Nelson, Division of Rheumatology, Mayo Clinic College of Medicine

K B Thomas, Division of Radiology, Mayo Clinic College of Medicine

This work was supported by the Division of Rheumatology, Mayo Clinic, Rochester, Minnesota, USA.

Correspondence to: Dr Tom Mason, Division of Rheumatology, Mayo Clinic, 200 First St SW, Rochester, MN 55905, USA; mason.thomas@ mayo.edu

Accepted 16 July 2004

\section{REFERENCES}

1 Peterson LS, Mason T, Nelson AM, O'Fallon WM, Gabriel SE. Juvenile rheumatoid arthritis in Rochester Minnesota 1960-1993. Is the epidemiology changing? Arthritis Rheum 1996;39:1385-90.

2 Brewer EJ, Bass J, Baum J, Cassidy J, Fink C, Jacobs J, et al. Current proposed revision of JRA criteria. Arthritis Rheum 1977:20:195-9.

3 Van der Heijde DMFM, van Leeuwen MA, van Riel PLCM, Koster AM, van't Hof, MA, van Rijswijk MH, et al. Biannual radiographic assessments of hands and feet in a three-year prospective follow-up of patients with early rheumatoid arthritis. Arthritis Rheum 1992;35:26-34.

4 Van der Heide A, Remme CA, Hofman DM, Jacobs JWG, Biilsma JWJ. Prediction of progression of radiologic damage in newly diagnosed rheumatoid arthritis. Arthritis Rheum 1995;38:1466-74.

5 Larsen A, Thoen J. Hand radiography of 200 patients with rheumatoid arthritis repeated after an interval of one year. Scand J Rheumatol 1987; 16:395-401

6 Sharp JT, Wolfe F, Mitchell DM, Bloch DA. The progression of erosion and joint space narrowing scores in rheumatoid arthritis during the first twenty-five years of disease. Arthritis Rheum 1991;34:660-8.

7 Resnick D, Niwayama G. Rheumatoid arthritis. In: Resnick D, ed. Diagnosis of bone and joint disorders, 3rd ed. Philadelphia: WB Saunders, 1995:866-970.

8 Paget SA. Treatment of rheumatoid arthritis. In: Klippel JH, Weyand CW, Wortman RL, eds. Primer on the rheumatic diseases, 11 th ed. Atlanta: Arthritis Foundation, 1997:68-174

9 Poznaski AK, Hernandez RJ, Guire KE, Bereza U, Garn SM. Carpal length in children: a useful measurement in the diagnosis of rheumatoid arthritis and some congenital malformation syndromes. Radiology 1978;129:661-8.

10 Magni-Manzoni S, Rossi F, Pistorio A, Temporini F, Viola S, Beluffi G, et al. Prognostic risk factors for radiographic progression, radiographic damage, and disability in juvenile idiopathic arthritis. Arthritis Rheum 2003:48:3509-17.

11 Fuchs HA, Sargent JS. Rheumatoid arthritis: the clinical picture. In: Koopman WJ, ed. Arthritis and allied conditions, 13th ed. Philadelphia: Williams and Wilkins, 1997:1041-70.

12 Mason T, Reed AM, Nelson AM, Thomas KB, Patton A, Hoffman AD, et al. Frequency of abnormal hand and wrist radiographs at the time of diagnosis of polyarticular juvenile rheumatoid arthritis. J Rheumatol 2002;29:21 14-218.

13 Oen K, Reed M, Malleson P, Cabral D, Petty R, Rosenberg A, et al. Radiologic outcome and its relationship to functional disability in juvenile rheumatoid arthritis (JRA). J Rheumatol 2003;30:832-40.

14 Van Rossum MAJ, Zwinderman AH, Boers M, Dijkmans BAC, van Soesbergen RM, Fiselier TJW, et al. Radiologic features of juvenile idiopathic arthritis. Arthritis Rheum 2003;48:507-15. 